\title{
Focalização e Cobertura do Programa de Aquisição de Alimentos (PAA): avaliação de sua eficácia nas regióes brasileiras ${ }^{1}$
}

\author{
Rafael Junior dos Santos Figueiredo Salgado ${ }^{2}$, Edson Arlindo Silva ${ }^{3}$, \\ Marco Aurélio Marques Ferreira ${ }^{4}$, Wellington Alvim Cunha ${ }^{5}$ e \\ Luana Ferreira dos Santos ${ }^{6}$
}

Resumo: A formulaçãoe a implementação de políticas públicas e programas governamentais têm ganhado destaque no cenário da administração pública brasileira; porém, ainda são escassas e incipientes as iniciativas voltadas à avaliação de políticas. Neste trabalho, objetivou-se avaliar a eficácia do Programa de Aquisição de Alimentos (PAA), classificando os municípios brasileiros em relação ao nível de propensão à execução desta política pública. Para isso, foram elaborados dois indicadores compostos por sete variáveis que englobassem tanto a propensão de insegurança alimentar da população local quanto a necessidade de acesso ao mercado por parte da agricultura familiar local. Posteriormente, foi estimado o nível de cobertura do programa nas regiões brasileiras. Foram utilizadas as técnicas estatísticas multivariada Análise de Cluster para identificar agrupamentos e Análise de Discriminante para validá-los. Foi possível identificar quatro grupos de demanda do programa com características distintas significativamente validados pela Análise Discriminante. O grupo com maior demanda das ações do programa é formado por municípios com expressiva presença de agricultores familiares, baixo dinamismo econômico e alto risco de segurança alimentar, e estão localizados majoritariamente nas regiões Nordeste e Norte. Constatou-se que o PAA tem concentrado suas ações nestes municípios; porém, o nível de cobertura do programa ainda é incipiente. Demonstrou-se, também, que a taxa de cobertura por regiões ainda é desigual, indicando que algumas regiões têm sido preteridas, com destaque para o Norte e o Nordeste.

Palavras-chaves: Programa de Aquisição de Alimentos, avaliação, políticas públicas, agricultura familiar, análise de cluster.

1. Data de submissão: 22 de setembro de 2015. Data de aceite: 20 de julho de 2017.

2. Universidade Federal de Viçosa. Viçosa, Minas Gerais, Brasil. E-mail: rafa.salgad@gmail.com

3. Universidade Federal de Viçosa. Viçosa, Minas Gerais, Brasil. E-mail: edsonsilva@ufv.br

4. Universidade Federal de Viçosa. Viçosa, Minas Gerais, Brasil. E-mail: marcoufv1@gmail.com

5. Universidade Federal de Viçosa. Viçosa, Minas Gerais, Brasil. E-mail: wellingtonalvimcunha@gmail.com

6. Universidade Federal de Viçosa. Viçosa, Minas Gerais, Brasil. E-mail: luana_agronegocio@hotmail.com 
Abstract: Conception and the practice of public policies and government programs have been highlighted in the Brazilian public administration. However, the initiatives to evaluate policies are still rare and incipient. We aim to evaluate the efficiency of food acquisition Program (PAA), classifying the Brazilian municipalities according to the level of tendency to implement this public policy. In order to do this, two indicators with seven variables were elaborated that incorporates both the propensity to food insecurity from local population and local familiar agriculture access to the market. Moreover, we estimated the coverage level of the program in Brazilian regions. We used multivariate statistical techniques Cluster analysis to identify groups and Discriminant analysis to validate them. As result, it was possible to identify four groups of program demand with distinct characteristics significantly validated by discriminant analysis. The group with highest demand of program actions is formed by municipalities with significant presence of family farmers, low economic dynamism and high risk of food security. They are mostly located in the Northeastern and Northern areas. Interestingly, we noted that the PAA has focused its actions in these municipalities. However, the level of coverage of the program is still incipient. Finally, the coverage rate based on regions is still uneven, indicating that some regions have been rejected, especially in North and Northeast of Brazil.

Key-words: Food Acquisition Program, evaluation, public policy, family agriculture, cluster analysis.

Classificação JEL: Q18.

DOI: http://dx.doi.org/10.1590/1234-56781806-94790550403

\section{Introdução}

A agricultura familiar vem ganhando espaço nos debates sobre a construção de políticas públicas no Brasil, que mostram a importância dessa categoria social para um desenvolvimento socioeconômico mais equilibrado e sustentável no meio rural. Segundo Müller, Silva e Schneider (2012), a elaboração de políticas públicas, que têm como foco a agricultura familiar, contribuiu para a legitimação das ações do Estado neste segmento e o reconhecimento social da importância da categoria social na sociedade brasileira.

Em contraste com a incontestável importância econômica e social da agricultura familiar, ela historicamente foi esquecida pelas principais políticas agrícolas (SOUZA e BARBE, 2014). Os investimentos públicos são escassos ou de difícil acesso aos agricultores familiares, principalmente pelo fato de as políticas agrícolas, comerciais e cambiais serem direcionadas aos produtores patronais (CORRÊA, 2008; BUAINAIN, ROMERO e GUANZIROLI, 2003; DELGADO, 2003).

Essa realidade começou a mudar a partir da década de 1990. As primeiras ações nacionais voltadas para a agricultura familiar resultaram da pressão de movimentos sociais ligados aos sindicatos rurais, culminando no desenvolvimento do Programa Nacional de Fortalecimento da Agricultura Familiar (Pronaf). A partir dessas ações, a agricultura familiar passa a ser vista pelo Estado como segmento estratégico para a produção de matérias-primas, geração de emprego e renda e abastecimento alimentar das populações (GRAZIANO DA SILVA, DEL GROSSI e DE FRANÇA, 2012). De fato, Abramovay (2007) aponta que a agricultura familiar seria o espaço para onde convergem desenvolvimento e equidade.

Embora o Pronaf tenha como público-alvo esta categoria social, e sua importância ser inquestionável, seu desempenho tem sido questionado por não levar em conta as especificidades da agricultura familiar e a diversidade de experiências de produção existentes (SOUZA e BARBE, 2014). Esta política tem enfrentado dificuldades de implementação junto aos agricultores mais vulneráveis e menos descapitalizados (ANDREWS, 2004), beneficiando, principalmente, as unidades familiares de produção em melhores condições socioeconômicas, localizadas nas regiões Sul e Sudeste, e promovendo o cultivo de produtos competitivos no mercado internacional, tais como o milho e a soja (GRISA e SCHNEIDER, 2014).

Diante desta realidade, aliadas à transição na esfera federal nos anos 2002/2003, houve a necessidade de criação de instrumentos governamentais para formar um novo mercado para a produção agrícola de base familiar e/ou para incluir os agricultores familiares, principalmente os mais fragilizados economicamente, aos mecanismos formais de apoio à 
produção agrícola (SIMÃO, SILVA e SILVEIRA, 2014; SILVA et al., 2012; IPC-IG, 2013). Como resposta a esta necessidade, criou-se o Programa de Aquisição de Alimentos (PAA) em 2003, uma das ações estruturantes do Programa Fome Zero, por meio da Lei Federal n. 10.696. O PAA advém de uma tendência recente de programas e políticas públicas no Brasil, com nítido recorte social (SIMÃO, SILVA e SILVEIRA, 2014; GRISA e SCHNEIDER, 2014). Com o PAA, além de um segmento estratégico para geração de renda e emprego, a agricultura familiar adquire papel importante na manutenção da segurança alimentar e nutricional do País (BECKER e SACCO DOS ANJOS, 2010).

Apesar de enfatizar a importância do PAA para a agricultura familiar, diversos estudos têm apontado limitações em sua implementação. Dentre elas, destacam-se a limitada e insuficiente abrangência e atuação do programa (LEMOS e LIMA, 2014; HESPANHOL, 2013); desigualdade de distribuição regional dos recursos (SIMÃO, SILVA e SILVEIRA, 2014); falta de articulação com outros programas direcionados à agricultura familiar (MARQUES, MOAL e ANDRADE, 2014); dificuldades relacionadas à operacionalização do programa e atraso na liberação dos recursos (SALGADO e DIAS, 2013; CERQUEIRA, ROCHA e COELHO, 2006), dentre outras.

Estes gargalos evidenciam a importância e necessidade do continuo monitoramento e avaliação de políticas, programas e projetos públicos. Esse processo tem ganhado notoriedade e relevância no setor público brasileiro nos últimos anos. Entretanto, Simão, Silva e Silveira (2014) e Carden e Alkin (2012) evidenciam que ainda são incipientes ou escassas as iniciativas voltadas para o processo de avaliação de políticas públicas e programas governamentais, apesar de observar-se crescimento na formulação e implementação destes. Isto ocorre em virtude da ausência de uma sistematização de dados e informações da gestão pública brasileira que dificultam e, às vezes, impossibilitam a mensuração dos resultados e o monitoramento das ações e dos programas governamentais (SIMÃO, SILVA e SILVEIRA, 2014).

Uma das formas de avaliação de políticas públicas sociais tem sido a utilização de mecanismos de focalização, que é geralmente justificada por uma questão de eficiência na alocação de recursos, o que significa concentrar um orçamento limitado nos mais necessitados (COADY, GROSH e HODDINOTT, 2004). Pode-se con- ceituar focalização como um instrumento de seleção ou processo que possibilita a seleção e a focalização de políticas públicas em determinados grupos, regiões, problemas (MIRANDA e COSTA, 2007). Um programa social é dito eficiente no critério de focalização quando define com clareza seu público-alvo. Portanto, a focalização pode ser vista como um instrumento para aumentar os efeitos sobre as populações mais demandantes, dada uma mesma quantidade de recursos disponíveis.

Partindo do pressuposto de que a avaliação não é, estritamente, a última fase do ciclo de uma política pública, mas sim uma fase constante no processo de elaboração e implementação de uma política, a contribuição e proposição deste trabalho é avançar na agenda de pesquisa de avaliações de políticas públicas, especificamente àquelas voltadas para a agricultura familiar. Devido à representatividade social e econômica para o campo e para a agricultura familiar, o presente trabalho buscou classificar os municípios brasileiros em relação à demanda do Programa de Aquisição de Alimentos e avaliar sua eficácia por meio da estimação do seu grau de cobertura, a exemplo do estudo de Simão, Silva e Silveira (2014), que analisou o grau de cobertura do programa no estado de Minas Gerais. Para isso, foram elaborados dois indicadores que representam a propensão à execução do programa. Especificamente, pretendeu-se verificar a focalização das atividades do PAA e, neste sentido, analisar se o programa tem obtido sucesso não só em incluir apenas os municípios mais demandantes, mas também cobrir todos os demandantes e, portanto, potencializando o fortalecimento da agricultura familiar e o processo de redução da pobreza e da desigualdade brasileira.

Em um primeiro momento, o artigo discute o processo de avaliação de políticas públicas, posteriormente apresenta-se o Programa de Aquisição de Alimentos. Em seguida, são listados os passos metodológicos que compuseram a pesquisa. Por fim, discorre-se sobre os resultados encontrados, finalizando com a exposição das conclusões.

\section{Processo de avaliação de políticas públicas}

Para fazer a avaliação de uma politica pública, primeiro deve-se entender o que é política pública. Conforme Teixeira (2002), políticas públicas podem 
ser definidas como diretrizes, princípios norteadores de ação do poder público, bem como regras e procedimentos para as relações entre poder público e sociedade. São políticas explicitadas, sistematizadas ou formuladas em documentos que orientam ações que normalmente envolvem aplicações de recursos públicos. Na prática, transformam-se em planos, programas ou projetos que trazem por si uma visão de determinado problema e uma proposição para enfrentá-lo. As políticas públicas têm como objetivo responder as demandas, principalmente dos setores marginalizados da sociedade, considerados vulneráveis. Visam, também, ampliar e efetivar direitos de cidadania, bem como promover o desenvolvimento, criando alternativas de geração de emprego e renda.

A avaliação de políticas públicas, especialmente programas sociais, tem ganhado importância crescente na esfera pública, nos centros de análise socioeconômica e universidades no Brasil, em decorrência da ampliação da escala e escopo da política social no país e, por conseguinte, pela necessidade de produção de informações mais específicas para o aprimoramento dos programas e ações governamentais (JANNUZZI, 2011). Avaliação pode ser entendida como a análise sistemática do processo ou dos resultados de um programa ou de uma política, tornando-se de fundamental importância para o seu aprimoramento (CUNHA, 2006).

Por intermédio de uma avaliação sistemática, contínua e eficaz, pode-se alcançar melhores resultados e propiciar uma melhor utilização e controle dos recursos aplicados. Pode-se também fornecer aos formuladores de políticas sociais e aos gestores de programas dados e informações importantes para o desenho de políticas mais consistentes e eficazes para a gestão pública, bem como guiar os tomadores de decisão, orientando-os quanto à continuidade, necessidade de correções ou mesmo suspensão de uma determinada política ou programa (COSTA e CASTANHAR, 2003).

Quanto às questões relacionadas aos objetivos de avaliação de políticas públicas, podem-se distinguir três modalidades: a eficiência ou processos, que está relacionada à otimização dos recursos empregados, no qual se utiliza os recursos disponíveis em busca do melhor resultado possível; eficácia ou metas e objetivos, que mede o grau em que o programa atinge seus objetivos e metas; efetividade ou impacto, indicando se o projeto tem efeitos positivos no ambiente externo em que interveio em termos técnicos, econômicos, socioculturais, institucionais e ambientais (COSTA e CASTANHAR, 2003; CARVALHO, 2003).

Este trabalho tem a avaliação de eficácia como foco, pois possui como preocupação central investigar se o PAA tem atingido suas metas e objetivos. A eficácia é uma dimensão do processo de desenvolvimento e implementação de um programa. O papel deste tipo de avaliação é buscar o aprimoramento do programa durante o processo de sua elaboração, isto é, esta avaliação pode ser usada para testar, rever e redefinir o programa de forma global ou sem seus diversos componentes (FARIA, 2006). Dessa forma, este estudo visou aferir se o programa está sendo implementado de acordo com as diretrizes básicas concebidas para sua execução e se têm atingido os municípios considerados mais demandantes. Segundo Arretche (1998), a avaliação de eficácia é o tipo de avaliação normalmente aplicado nas avaliações de políticas públicas. Isto se deve por ser a mais factível e menos custosa de ser realizada.

A maior dificuldade atual na avaliação de programas e políticas públicas é o acesso às informações necessárias para o acompanhamento dos resultados e do impacto que tais ações desempenham junto aos segmentos sociodemográficos ou nas comunidades por eles focalizadas (JANNUZI, 2005). Um eficiente medidor da eficácia das ações governamentais no âmbito das políticas públicas encontra-se no uso de indicadores. Se bem empregados, os indicadores podem enriquecer a interpretação empírica da realidade social e orientar de forma mais competente a análise, formulação e implementação de políticas públicas, além de possibilitar um melhor acompanhamento, por parte da sociedade, dos resultados alcançados (JANNUZZI, 2002; REZENDE e JANNUZZI, 2008).

\section{O Programa de Aquisição de Alimentos}

O Programa de Aquisição de Alimentos foi instituído pelo art. 19 da Lei n. 10.696, de 2 de julho de 2003. Tem como finalidade apoiar os agricultores familiares, por meio da aquisição de alimentos de sua produção com dispensa de licitação (BRASIL, 2015a). Foi o primeiro programa de aquisição institucional de alimentos da agricultura familiar criado pelo Brasil, com três objetivos principais: (i) apoiar os agricultores fami- 
liares por meio do incentivo à produção e da garantia de acesso ao mercado; (ii) distribuir alimentos para pessoas em insegurança alimentar e nutricional e (iii) construir estoques estratégicos de produtos agrícolas (SWENSSON, 2015).

Foi concebido para apoiar pequenos agricultores em um dos aspectos mais difíceis do processo produtivo: garantir acesso ao mercado para os produtos que eles produzem. O programa permite que os agricultores vendam seus produtos a instituições públicas federais, estaduais e municipais, sem a necessidade de licitação, conectando grandes fontes previsíveis de demanda institucional de produtos agrícolas aos agricultores familiares, reduzindo riscos, incentivando a melhoria na qualidade do produto, aumentando a renda e reduzindo a pobreza no meio rural. Ademais, o PAA também atua na aquisição de produtos para armazenamento, regulação dos preços de mercado e assistência a grupos de indivíduos em insegurança alimentar e nutricional (IPC-IG, 2013; MILHORANCE e GABAS, 2015).

Desde sua criação, vários estudos e avaliações já foram realizados, subsidiando as mudanças promovidas no PAA (TRICHES e GRISA, 2015). Estes trabalhos apontam para as contribuições do programa na sustentação de preços e garantia e melhoria da renda (CERQUEIRA, ROCHA e COELHO, 2006; DIAS e ROCHA, 2015; ORTEGA, JESUS e SÓ, 2006), fortalecimento da política global de combate à fome e promoção de estoques estratégicos (MATTEI, 2007), criação e ampliação de mercados para o segmento da agricultura familiar (SILVA, ROSA e AMODEO, 2014), no incremento da produção e diversificação (HESPANHOL, 2013; LEAL, 2015), na melhoria da qualidade de vida (SALGADO e SILVA, 2013; MOTA et al., 2014), entre outras.

Em que pesem as contribuições do PAA, estudos têm demostrado que o Programa ainda apresenta limitações que dificultam sua operacionalização e melhor desempenho. A literatura tem apontado que os principais limites são a divulgação restrita dessa política pública (SPAROVEK et al., 2007), o desconhecimento por parte dos beneficiários dos objetivos e da dinâmica do programa (GOMES e BASTOS, 2007), inconformidades na seleção dos beneficiários (SPAROVEK et al., 2007; SOARES e BARROS e MAGALHÃES, 2007) e problemas de logística (atraso na liberação de recursos, dificuldades com o transporte dos produtos, obtenção da DAP, falta de assistência técnica, etc.) (CORDEIRO, 2007).

Observa-se que, ao mesmo tempo em que o PAA apresenta inovações institucionais como política pública, ao criar instrumentos de crédito com garantia de compra ("compra antecipada", "garantia de compra", "compra direta local" etc.) dirigida especificamente para a agricultura familiar, ainda podem haver inconformidades relativas às suas atribuições iniciais. Ademais, as ações governamentais do programa têm concentrado a aplicação de recursos estritamente em municípios com expressiva insegurança alimentar, deixando de lado aspectos relacionados à provisão do fomento produtivo à agricultura familiar (SIMÃO, SILVA e SILVEIRA, 2014; DELGADO, CONCEIÇÃO e OLIVEIRA, 2006). Dessa forma, estudos que objetivam avaliar o programa, por meio de seu grau de cobertura e focalização, são pertinentes para contribuir com sua contínua melhoria e também subsidiar futuras decisões por parte dos gestores públicos, além de evidenciar novos caminhos a outras pesquisas. Ademais, pelo seu escopo nacional, este trabalho pode servir como norteador para a avaliação e acompanhamento de políticas que visem ao desenvolvimento rural em outros países interessados na implementação de programas similares.

\section{Metodologia}

O presente trabalho pautou-se em uma abordagem quantitativa. Foram elaborados indicadores que representassem o risco de insegurança alimentar e necessidade de acesso ao mercado dos agricultores familiares. Fez-se uso de métodos quantitativos para verificar a confiabilidade e consistência dos constructos e proxys analisadas por meio do Teste Alpha de Cronbach. Posteriormente, utilizou-se a Técnica Estatística Multivariada Análise de Clusters para agrupar as cidades por homogeneidade de avaliação. Adicionalmente, foi utilizada a técnica de Análise Discriminante Múltipla como forma de corroborar a validação dos grupos que foram encontrados. Por fim, também foram utilizadas as Tabelas Cruzadas e a Análise de Frequência para caracterizar e analisar os grupos formados.

Foram coletados dados de todos os 5.565 municípios brasileiros, excluindo-se aqueles em que havia falta 
de dados. Posteriormente, identificaram-se todos os municípios brasileiros que comercializaram seus produtos via Programa de Aquisição de Alimentos (PAA) entre janeiro e dezembro de 2013, em termos de número de agricultores beneficiados e volume de recursos financeiros repassados aos agricultores. Estas informações foram obtidas de dados secundários, por meio da plataforma PAA Data da Secretaria de Informação e Gestão do MDS do governo federal (BRASIL, 2015b). Optou-se por utilizar dados de 2013, pois, quanto mais atuais os dados, maior a representatividade da realidade observada. Quanto às modalidades analisadas, foram as seguintes: aquelas operacionalizadas pela Conab (Compra Direta da Agricultura Familiar, Formação de Estoque pela Agricultura Familiar e Compra com Doação Simultânea), e as operacionalizadas por estados e municípios (Compra com Doação Simultânea e Incentivo à produção e Consumo de Leite - PAA Leite).

O PAA teve sua concepção articulada em torno de duas políticas públicas: a política agrícola e a política de segurança alimentar. Em virtude da concentração dos objetivos do PAA nestas duas linhas principais, quais sejam, propiciar acesso ao mercado aos agricultores familiares e diminuir a vulnerabilidade alimentar, escolheu-se pela elaboração de constructos ou dimensões que representassem de maneira mais fiel essas duas linhas, de forma a representar a real situação do programa no contexto analisado. Estes indicadores têm como objetivo conjunto elencar os municípios brasileiros com maior propensão à execução do programa e, desta forma, evidenciar as cidades mais demandantes em relação às duas principais linhas de atuação.

A propensão à execução do PAA foi composta de dois indicadores: um que representa o Risco de Insegurança Alimentar da população local e outro que representa a Necessidade de Acesso ao Mercado por parte dos agricultores familiares. Estes indicadores foram elaborados tomando por base os dados de todos os municípios brasileiros. A elaboração desses indicadores e as respectivas variáveis tiveram como base o trabalho de Simão, Silva e Silveira (2014).

O Quadro 1 apresenta as variáveis utilizadas para compor cada proxy, bem como o ano, descrição e a fonte dos dados:

Quadro 1. Variáveis utilizadas para compor os indicadores

\begin{tabular}{|c|c|c|c|}
\hline Proxy & Variável & Descrição & Fonte \\
\hline \multirow[t]{4}{*}{$\begin{array}{l}\text { Risco de Insegu- } \\
\text { rança Alimentar }\end{array}$} & $\begin{array}{l}\text { Índice de Desenvolvimento } \\
\text { Humano - Dimensão Educação } \\
\text { (IDHM-E) - } 2010\end{array}$ & $\begin{array}{l}\text { Índice sintético da dimensão Educação que } \\
\text { é um dos } 3 \text { componentes do IDHM. É obtido } \\
\text { através da média geométrica do subíndice de } \\
\text { frequência de crianças e jovens à escola, com } \\
\text { peso de } 2 / 3 \text {, e do subíndice de escolaridade } \\
\text { da população adulta, com peso de } 1 / 3 \text {. }\end{array}$ & $\begin{array}{l}\text { Atlas do Desenvolvimen- } \\
\text { to Humano do Brasil } \\
\text { (PNUD, 2013) }\end{array}$ \\
\hline & $\begin{array}{l}\text { Rendimento Domiciliar Médio per } \\
\text { capita Nominal - } 2010\end{array}$ & $\begin{array}{l}\text { Média das rendas domiciliares per capita das } \\
\text { pessoas residentes em determinado espaço } \\
\text { geográfico, no ano considerado. }\end{array}$ & \multirow[t]{2}{*}{$\begin{array}{l}\text { Censo Demográfico } \\
\text { (IBGE, 2010) }\end{array}$} \\
\hline & $\begin{array}{l}\text { Proporção População Fora da Si- } \\
\text { tuação de Extrema Pobreza - } 2010\end{array}$ & $\begin{array}{l}\text { Quantidade de moradores de domicílios } \\
\text { particulares permanentes que não estão em } \\
\text { situação de extrema pobreza. }\end{array}$ & \\
\hline & $\begin{array}{l}\text { Índice de Desenvolvimento da } \\
\text { Família (IDF) - Dimensão Dispo- } \\
\text { nibilidade de Recursos - } 2013\end{array}$ & $\begin{array}{l}\text { Representa a renda e a despesa familiar per } \\
\text { capita e as despesas relativas à alimentação } \\
\text { daquelas famílias inscritas no Cadastro Único } \\
\text { do governo federal. }\end{array}$ & $\begin{array}{l}\text { Ministério do Desenvol- } \\
\text { vimento Social (BRASIL, } \\
2013 \text { ) }\end{array}$ \\
\hline \multirow{3}{*}{$\begin{array}{l}\text { Necessidade } \\
\text { de Acesso ao } \\
\text { Mercado }\end{array}$} & $\begin{array}{l}\text { Estabelecimentos Pertencentes à } \\
\text { Agricultura Familiar - } 2006\end{array}$ & $\begin{array}{l}\text { Número municipal de estabelecimentos da } \\
\text { Agricultura Familiar (Lei n }{ }^{0} 11.326 \text { ) }\end{array}$ & $\begin{array}{l}\text { Censo Agropecuário de } \\
2006 \text { (IBGE, 2006) }\end{array}$ \\
\hline & $\begin{array}{l}\text { Proporção População Rural em Si- } \\
\text { tuação de Extrema Pobreza - } 2010\end{array}$ & $\begin{array}{l}\text { Quantidade de moradores de domicílios } \\
\text { particulares permanentes rurais em situação } \\
\text { de extrema pobreza. }\end{array}$ & $\begin{array}{l}\text { Censo Demográfico } \\
\text { (IBGE, 2010) }\end{array}$ \\
\hline & $\begin{array}{l}\text { Proporção Trabalhadores do Setor } \\
\text { Agropecuário - } 2010\end{array}$ & $\begin{array}{l}\text { Razão entre o número de pessoas de } 18 \text { anos } \\
\text { ou mais de idade ocupadas no setor agrope- } \\
\text { cuário e o número total de pessoas ocupadas } \\
\text { nessa faixa etária. }\end{array}$ & $\begin{array}{l}\text { Atlas do Desenvolvimen- } \\
\text { to Humano do Brasil } \\
\text { (PNUD, 2013) }\end{array}$ \\
\hline
\end{tabular}

Fonte: Elaborado pelos autores. 
As variáveis "IDHM-E" e "Rendimento per capita" foram escolhidas com base em estudos acerca da segurança e insegurança alimentar no Brasil: Segall-Corrêa et al. (2008); Hoffman (2013); Vianna e Segall-Corrêa (2008) e Oliveira et al. (2009) que indicaram que o rendimento per capita e a escolaridade, avaliada através da proxy IDHM-E, são apontados como fator importante na determinação da situação de segurança alimentar em que se encontram os moradores.

A disponibilidade de alimentos e as condições de infraestrutura determinam o acesso físico ao alimento; e o acesso econômico depende do poder de compra da família e do nível de preços de alimentos (THOMSON e METZ, 1998). Assim, Tweeten (1999) destaca que, diante da grande possibilidade de que os alimentos estejam disponíveis nos mercados mundiais para qualquer indivíduo com poder de compra, provavelmente a acessibilidade seja uma das maiores restrições à segurança alimentar. Portanto, optou-se pela variável IDF na sua dimensão Disponibilidade de Recursos por representar o dispêndio médio das famílias de baixa renda com alimentação. Esta dimensão evidencia a renda, a despesa familiar per capita e as despesas relativas à alimentação das famílias.

Segundo Hoffman e Kageyama (2007), extrema pobreza refere-se a pessoas com renda abaixo da linha de pobreza e cujo domicílio não possui água canalizada em nenhum cômodo, nem banheiro ou sanitário e nem luz elétrica, ou seja, encontra-se em estado de privação dos três equipamentos básicos. Com base na definição de extrema pobreza e no estudo de Vianna e Segall-Corrêa (2008), que mostraram que as condições de moradia estão fortemente associadas à situação de insegurança alimentar, e que, devido à correlação com a renda, atinge fortemente a população pobre, optou-se por utilizar a variável "Proporção da População Fora da Situação de Extrema Pobreza" por estar fortemente relacionado com a situação de segurança alimentar.

Para Buainain (2006), o acesso ao mercado é um ponto fundamental para o sucesso do empreendimento rural familiar. Buscando representar este fator, as variáveis "Estabelecimentos Pertencentes à Agricultura Familiar" e "Proporção População Rural em Situação de Extrema Pobreza" foram escolhidas por representarem os municípios com maior presença do público alvo do Programa de Aquisição de Alimentos: agricultores familiares e população rural em situação de extrema pobreza. Além de permitir o acesso ao mer- cado institucional, o programa deve focalizar aqueles agricultores mais vulneráveis socioeconomicamente, promovendo a geração de renda e redução da extrema pobreza rural.

A variável "Proporção de Trabalhadores do Setor Agropecuário" foi selecionada por representar a importância do PAA para a geração e manutenção de empregos no meio rural, uma vez que, além de ser importante fornecedora de alimentos, estudos comprovam a importância da agricultura familiar também como principal fonte de ocupação da força de trabalho no meio rural brasileiro (GUANZIROLI et al., 2001; KAGEYAMA, BERGAMASCO e OLIVEIRA, 2013), e o Censo Agropecuário de 2006 registrou 12,3 milhões de pessoas vinculadas à agricultura familiar, o que representa $74,4 \%$ do pessoal ocupado, enquanto os estabelecimentos não familiares ocupavam 4,2 milhões de pessoas, correspondendo a $25,6 \%$ da mão de obra ocupada.

Dentre as medidas de consistência interna de constructos disponíveis na literatura, a recomendada é o coeficiente Alfa de Cronbach, cujo resultado varia de 0 a 1 , que é baseado no seu número de itens e na intercorrelação dos itens. É um índice utilizado para medir a confiabilidade do tipo de consistência interna de uma escala, ou seja, para avaliar a magnitude em que os itens de um instrumento estão correlacionados (HAIR JUNIOR et al., 2005). Quanto à confiabilidade da proxy Risco de Insegurança Alimentar, obteve-se um Alfa de 0,902, o que é considerado excelente pela literatura. Já a proxy Necessidade de Acesso ao Mercado obteve Alfa de Cronbrach de 0,601 que, apesar de baixo, é considerado adequado aos objetivos. Diante da elaboração destas dimensões procede-se a elaboração dos índices de Risco de Insegurança Alimentar e Necessidade de Acesso ao Mercado para todos os municípios brasileiros.

Posteriormente, utilizou-se a Análise de Cluster para agrupar os municípios em grupos homogêneos para, posteriormente, classificá-los quanto ao nível de propensão à execução do programa, especialmente para fins de comparação da taxa total de cobertura das ações do programa em cada uma das grandes regiões brasileiras. A Análise de Cluster consiste numa análise exploratória de dados que pretende organizar diferentes variáveis para que o grau de associação entre duas variáveis seja máximo, se pertencerem ao mesmo grupo, e mínimo, em caso contrário. O objetivo 
básico desta técnica consiste em descobrir agrupamentos naturais de variáveis que apresentem, simultaneamente, coesão interna e separação externa. Esta técnica agrupa os municípios com características semelhantes, indicando os municípios mais e menos demandantes da execução da política pública do PAA. Essa análise tem sido utilizada em diferentes contextos e em muitas áreas por diversos pesquisadores (COSTA et al., 2013; KOUTSOU, PARTALIDOU e PETROU, 2011; LLANILLO et al., 2006; CASTEL et al., 2010), o que reflete seu apelo mais amplo e sua utilidade como técnica estatística.

A primeira fase da Análise de Cluster divide a amostra total em subgrupos menores. Nesta fase, definem-se as distâncias entre os objetos que estão aglomerados. Utilizou-se a medida Distância Euclidiana, mais comumente utilizada na literatura e aconselhada quando as variáveis do estudo são quantitativas (GIUDICI e FIGNI, 2009). Além de estabelecer qual a forma de cálculo da distância entre observações, é ainda necessário estabelecer a forma de cálculo da distância entre grupos. Dois tipos de procedimentos são utilizados para agrupar objetos semelhantes: hierárquico e não hierárquico. Ambos os tipos tentam maximizar as diferenças (distâncias) entre conglomerados em relação à variação dentro deles.

Num primeiro momento, utilizou-se o conglomerado hierárquico para identificar o número de grupos a considerar. Este método permite obter uma família de partições, associadas com subsequentes níveis de agrupamento entre as observações, que podem ser representadas graficamente por dendogramas. Isso pode ser feito utilizando a abordagem aglomerativa ou uma abordagem divisória, sendo a abordagem aglomerativa amplamente utilizada. Existem várias opções para se calcular conglomerados aglomerativos, e este estudo utilizou o método Ward. Segundo Hair Junior et al. (2005), este método é muito popular e, mais do que outros métodos, tende a resultar em conglomerados com aproximadamente o mesmo número de objetos, por isso é o mais recomendado. A escolha deste método vai de encontro com a medida de distância utilizada, uma vez que as escolhas de um método de aglomeração e de uma medida de distância estão inter-relacionadas.

Identificado o número de grupos a reter pelo método hierárquico, aplicou-se o método não hierárquico K-means mais indicado para amostras maiores
( $\mathrm{N}>1.000)$, caso deste estudo, já que não computa a matriz de proximidade de distâncias/similaridade entre todos os casos observados (GARSON, 2014). Este método baseia-se diretamente na escolha antecipada de um número de grupos que conterão todos os casos. Segundo Nunes e Barros (2012), procede-se, em seguida, a uma divisão de todos os casos pelo k grupos pré-estabelecidos e a melhor partição dos n casos será aquela que otimizar o critério escolhido.

Uma vez determinado o número de conglomerados a reter, verifica-se que os grupos são, na verdade, estatisticamente diferentes e teoricamente significativos. Nesta fase foi utilizada a Análise de Discriminante para validar a Análise de Cluster. Segundo Hair Junior et al. (2005), a Análise Discriminante encontra ampla aplicação em situações nas quais o objetivo principal é identificar o grupo a qual um objeto pertence.

\section{Resultados e discussão}

Os dados coletados para os 5.565 municípios brasileiros foram subdivididos por regiões e estados. As variáveis coletadas contemplam características das dimensões Risco de Insegurança Alimentar e Necessidade de Acesso ao Mercado. Para evitar que alguma medida de semelhança/distância refletisse o peso das variáveis de valores maiores e dispersão, já que as variáveis se apresentam definidas em diferentes escalas de medida, procedeu-se à padronização prévia. Efetuada a padronização das variáveis para cada indicador, estas foram somadas de forma que compusessem um único score por dimensão.

O método hierárquico de Cluster consiste em uma série de sucessivos agrupamentos ou sucessivas divisões de objetos, onde os objetos são agregados ou desagregados. Desta forma, pelo método hierárquico de agrupamento, ou mais especificamente a técnica de Ward, realizou-se o teste de 2 a 4 Clusters. Porém, aquele que atendeu às características das variáveis e ao aporte teórico deveu-se à formação de quatro grupos.

Após a determinação do número de grupos, utilizou-se o K-means para aperfeiçoar o resultado. Como variável dependente utilizou-se os clusters formados por meio da análise de agrupamentos. As variáveis independentes foram representadas pelas dimensões Risco de Insegurança Alimentar e Necessidade de Acesso ao Mercado, utilizadas para formar os agrupamentos. 
Posteriormente, para confirmação dos clusters, foi aplicada a técnica de Análise de Discriminante para validar os grupos encontrados. Foram obtidas duas funções discriminantes distintas denominadas F1 e F2. Somente a primeira apresentou alta capacidade de demonstração das diferenças entre os grupos, com valor de $98,9 \%$. A segunda função apresentou um poder discriminante que explica apenas 1,1\% das variâncias entre os grupos. Neste caso, obteve-se $100 \%$ de explicação da variância total, com essas duas variáveis comprovando que as classificações dos grupos foram realizadas corretamente. Torna-se importante mencionar que se obteve valor de 0,00 para o lambda de Wilks, demonstrando, assim, que as médias dos grupos são consideravelmente diferentes.

Quanto à mensuração do erro, este compreendeu apenas 1,8\% dos casos, em que as observações foram agrupadas de maneira atípica às demais, sendo assim, a precisão da classificação (razão de sucesso) é de 98,2\% . Logo, torna-se possível afirmar que o resultado dessa pesquisa compreende a identificação de quatro clusters distintos. Após esse procedimento, a análise descritiva dos indicadores considerados torna-se um instrumento para categorizar as unidades de cada Cluster. Objetivando interpretar os grupos formados, para verificar a combinação dos indicadores que levou à construção de cada agrupamento, foi realizada a análise dos indicadores. Em virtude da combinação dos indicadores, as cidades foram divididas em quatro grupos em função da demanda por ações do Programa de Aquisição de Alimentos: Muito Demandante, Demandante, Pouco Demandante, Nada Demandante (Tabela 1).

As Tabelas 2 e 3 relacionam as características predominantes nos quatro grupos formados. O Agrupamento 1 é formado por 794 municípios caracterizados como Muito Demandantes do Programa de Aquisição de Alimentos. Os municípios desse grupo estão localizados majoritariamente na região Nordeste $(83 \%)$ e $15 \%$, na região Norte, o que evidencia a necessidade de focalização e ampliação desta política para estas duas regiões que concentram $66 \%$ do total de pessoas pobres domiciliadas no meio rural brasileiro e $81 \%$ da população rural extremamente pobre (MATTEI e MALUF, 2011), indicando que as condições de vida ainda são precárias, há altíssimo risco de insegurança alimentar e alta necessidade de acesso a mercado por parte dos agricultores familiares. Este grupo tem os piores indicadores de insegurança alimentar, e os municípios possuem, em média, o maior número de estabelecimentos da agricultura familiar. Aproximadamente $49 \%$ da população rural deste grupo vive em situação de extrema pobreza, os municípios possuem trabalhadores predominantemente do setor agropecuário (53,9\%). Deve-se ressaltar, também, que apenas $2 \%$ dos municípios muito demandantes pertencem à região Sudeste, e 0,3\% , à região Sul, regiões que historicamente têm recebido considerável aporte do PAA.

O Grupo 2 é formado por 1.395 municípios caracterizados como Demandantes do Programa de Aquisição de Alimentos. Desse total, 68\% estão localizados na região Nordeste, $14 \%$, na região Norte e $14 \%$, no Sudeste. São caracterizados por municípios de baixa renda per capita, baixo Índice de Desenvolvimento Humano na educação, têm, em média, aproximadamente $24 \%$ da população em situação de extrema pobreza, grande parte dos trabalhadores estão ocupados no setor agropecuário (em média, 41,5\%). Possui o segundo maior número médio de estabelecimentos da agricultura familiar, além de apresentar grande parcela da população rural em situação de extrema pobreza $(29,5 \%)$. Desta forma, podem ser caracterizados como municípios onde políticas públicas voltadas para a agricultura familiar e combate à fome e pobreza devem ser implementadas. A análise das variáveis indica, ainda, que são municípios em que a população possui alto risco de insegurança alimentar e há, também, necessidade de acesso ao mercado por parte dos agricultores.

Tabela 1. Análise de desempenho e classificação dos grupos

\begin{tabular}{lcc}
\hline \multicolumn{1}{c}{ Grupo } & Necessidade de Acesso ao Mercado & Risco de Insegurança Alimentar \\
\hline Grupo 1 - Muito Demandante & Muito Alto & Muito Alto \\
Grupo 2 - Demandante & Médio & Alto \\
Grupo 3 - Pouco Demandante & Médio & Baixo \\
Grupo 4 - Nada Demandante & Baixo & Muito Baixo \\
\hline
\end{tabular}

Fonte: Resultados da pesquisa. 
Tabela 2. Média das variáveis para os quatro grupos formados

\begin{tabular}{lcccc}
\hline \multirow{2}{*}{\multicolumn{1}{c}{ Variáveis }} & \multicolumn{3}{c}{ Agrupamentos } \\
\cline { 2 - 5 } & M. Demandante & Demandante & P. Demandante & N. Demandante \\
\hline IDH-E & 0,444 & 0,491 & 0,565 & 0,654 \\
Renda Per Capita & 194,3 & 257,1 & 433,5 & 634,5 \\
População Fora Extrema Pobreza & 64,9 & 77,8 & 92,4 & 97,4 \\
Ocupados no Setor Agropecuário & 53,9 & 41,5 & 37,3 & 22,0 \\
Estabelecimento Agricultura Familiar & 1850,2 & 816,1 & 649,8 & 418,5 \\
População Rural em Extrema Pobreza & 48,8 & 29,5 & 11,3 & 3,6 \\
IDF & 0,506 & 0,556 & 0,652 & 0,714 \\
\hline
\end{tabular}

Fonte: Resultados da pesquisa.

Tabela 3. Divisão dos grupos por região

\begin{tabular}{lccccccccc}
\hline \multirow{2}{*}{ Regiões } & \multicolumn{2}{c}{ Muito Demandante } & \multicolumn{2}{c}{ Demandante } & \multicolumn{2}{c}{ Pouco Demandante } & \multicolumn{3}{c}{ Nada Demandante } \\
\cline { 2 - 9 } & Total & $\%$ & Total & $\%$ & Total & $\%$ & Total & $\%$ \\
\hline Centro-Oeste & 3 & $0,4 \%$ & 34 & $2 \%$ & 225 & $15 \%$ & 204 & $11 \%$ & 466 \\
Nordeste & 659 & $83 \%$ & 951 & $68 \%$ & 163 & $11 \%$ & 21 & $1 \%$ & 1794 \\
Norte & 117 & $15 \%$ & 191 & $14 \%$ & 124 & $8 \%$ & 17 & $1 \%$ & 449 \\
Sudeste & 13 & $2 \%$ & 189 & $14 \%$ & 545 & $36 \%$ & 921 & $49 \%$ & 1668 \\
Sul & 2 & $0,3 \%$ & 30 & $2 \%$ & 438 & $29 \%$ & 718 & $38 \%$ & 1188 \\
Brasil & 794 & & 1395 & & 1495 & & 1881 & 5565 \\
\hline
\end{tabular}

Fonte: Resultados da pesquisa.

O Grupo 3 é formado por 1.495 municípios que foram classificados como Pouco Demandantes. Dos municípios, $36 \%$ estão localizados na região Sudeste, $29 \%$, no Sul e 15\%, na região Centro-Oeste. Estes municípios possuem aproximadamente $8 \%$ da população em situação de extrema pobreza, e a população rural em extrema pobreza é um pouco maior, 11,3\%. Possuem IDH-E e IDF relativamente maiores em relações aos grupos 1 e 2 e têm, em média, 650 estabelecimentos da agricultura familiar por município e aproximadamente $37 \%$ dos trabalhadores trabalham no setor agropecuário. Apesar de este grupo de municípios ter indicadores socioeconômicos relativamente melhores, a análise dos dados indica que demandam políticas voltadas à agricultura familiar, pois possuem expressiva quantidade de estabelecimentos da agricultura familiar e grande parte da população ocupada trabalha no setor agropecuário.

O Agrupamento 4, denominado Nada Demandantes, é formado por 1.881 municípios, o maior grupo. Isso significa que a maioria dos municípios brasileiros possui muito pouca ou nenhuma demanda por ações do PAA. Este grupo é o que tem menor risco de insegurança alimentar e também o que possui menor demanda de acesso ao mercado por parte dos agricultores familiares, devido ao fato de, em média, apenas 3\% da população rural e da população urbana estarem em situação de extrema pobreza. Possui ainda, o menor número de estabelecimentos da agricultura familiar, a maior renda per capita dentre os grupos analisados, além da menor proporção de trabalhadores ocupados no setor agropecuário, $21 \%$. São municípios em que a atividade agropecuária não é forte e são mais desenvolvidos economicamente. Essas características ficam evidentes quando verifica-se que $49 \%$ destes municípios estão no Sudeste, e 38\%, na região Sul.

A Figura 1 apresenta a distribuição geográfica dos agrupamentos formados na Análise de Cluster. 
Figura 1. Distribuição dos diferentes agrupamentos por municípios brasileiros

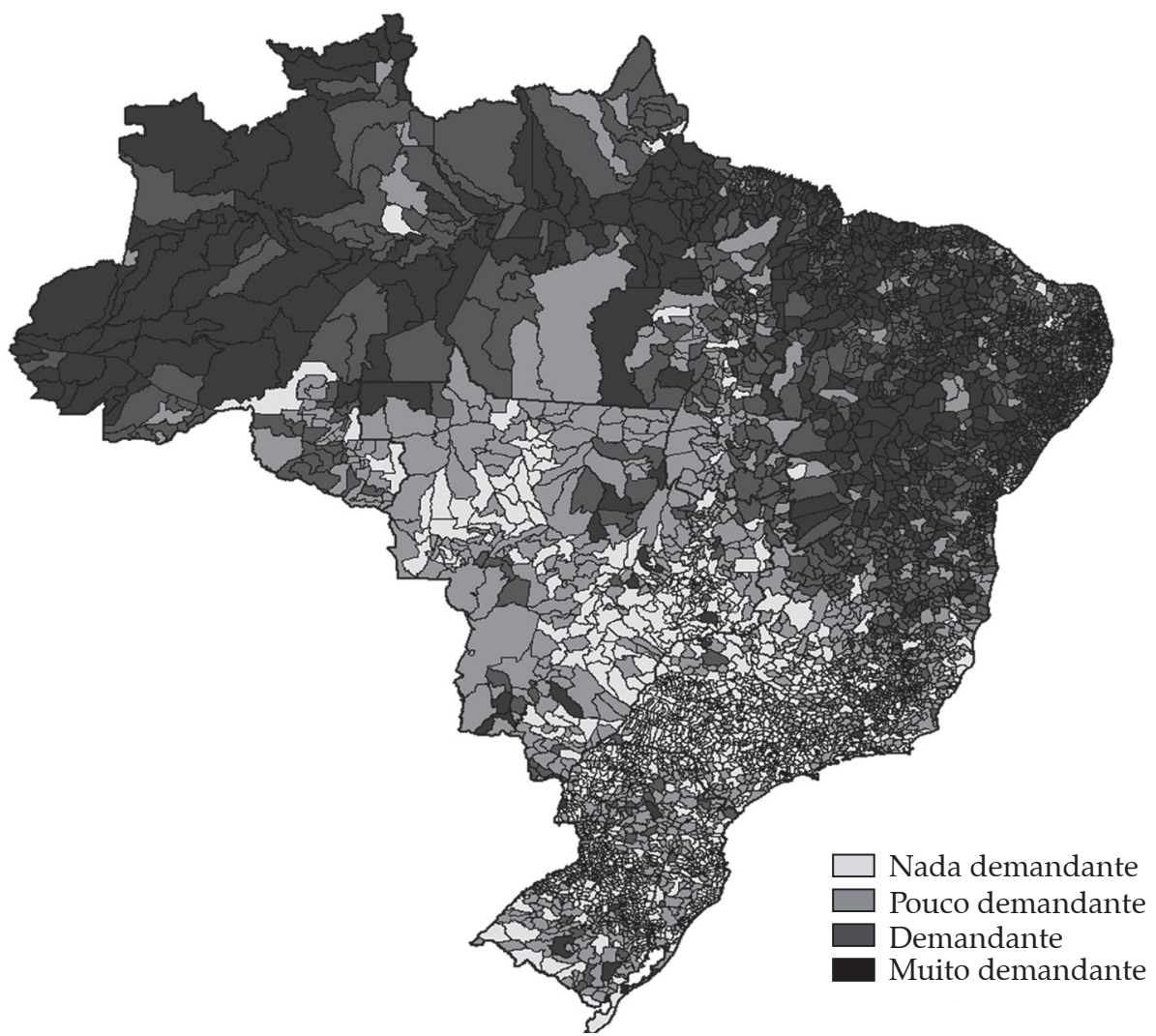

Fonte: Resultados da pesquisa.

Após análise dos agrupamentos, e com base nos grupos formados pela Análise de Cluster, que possibilitou criar informações preliminares imprescindíveis para a realização da avaliação e monitoramento do Programa de Aquisição de Alimentos, procedeu-se à avaliação da eficácia da operacionalização do programa no Brasil junto às regiões foco por meio da análise do grau de cobertura do programa. Inicialmente, verificou-se que, dos 5.565 municípios brasileiros, aproximadamente $20 \%$ (1.089) participaram do programa em 2013, nas modalidades analisadas. Desses municípios, a Análise de Cluster aponta para uma taxa de focalização de $58 \%$ (641 cidades), ou seja, a maioria dos agricultores familiares e da população em situação de insegurança alimentar beneficiados pelo PAA estão em cidades que apresentam demanda real de suas ações, classificados nos grupos de municípios demandantes e muito demandantes.

O maior grau de cobertura, proporcional aos agrupamentos retidos pela Análise de Cluster, concentra- -se nos municípios cuja demanda de execução é maior (Tabela 4). Estas constatações foram obtidas através da comparação percentual entre os municípios que participaram do programa e o percentual não participante em cada agrupamento para todas as cidades do Brasil. Os municípios que foram classificados como pouco ou nada demandantes apresentaram expressiva taxa de cobertura, de em média 13,5\%, evidenciando que parte considerável dos recursos do PAA está sendo direcionada para municípios com menor propensão à execução do programa. Trata-se de um número bastante elevado, pois aproximadamente $40 \%$ dos municípios que executaram o programa em 2013 foram classificados nestes agrupamentos. Estes resultados evidenciam que a execução do programa não depende apenas da indução do Estado, mas, principalmente, do capital social da agricultura familiar de cada região.

A presença de agricultores familiares mais organizados em sua produção, em associações e cooperativas e com maior interlocução com atores externos 
(extensão rural, gestores públicos etc.) induz maior acesso à informação por parte dos produtores ou de suas representações legais (CONAB, 2014) e, consequentemente, a maior acesso a mercados institucionais. Estudos têm apontado a divulgação restrita do programa como uma das suas principais limitações (DORETTO e MICHELLON, 2007; SPAROVEK et al., 2007; CORDEIRO, 2007). Conforme Cordeiro (2007), a pouca divulgação impede que um número maior de pessoas tenha acesso ao PAA, sendo que o principal veículo de tomada de conhecimento tem sido as redes sociais. Este aspecto tem sido uma das principais preocupações dos gestores, pois há descompasso entre o crescimento do número de agricultores que buscam acessar o PAA e a sua oferta orçamentária. Provavelmente, este seja um dos motivos que tenham limitado a divulgação (GRISA et al., 2010).

Apesar do resultado positivo no que concerne à taxa de focalização, observa-se que a taxa de cobertura nos municípios demandantes ainda é baixa. Este fato talvez esteja relacionado à reduzida execução do PAA em 2013, um ano verdadeiramente atípico, razão pela qual foram executados apenas $38 \%$ dos recursos em comparação a 2012. A falta de continuidade do programa é apontada como um fator limitador, haja vista que o PAA ainda é concebido como uma política de governo com ações localizadas e muitas vezes descontinuadas, e não uma ação de Estado (GRISA, et al., 2010). Ademais, a baixa execução pode ser atribuída a fatores diversos, a exemplo da seca ocorrida no Nordeste, envolvimento das Superintendências no programa Venda em Balcão, redução da demanda de Compra Direta, cancelamento da Formação de Estoque com liquidação física, remodelação dos normativos e outros fatores que, somados, culminaram neste desfecho (CONAB, 2014).

Os resultados parecem favoráveis à eficácia do Programa, uma vez que a maior concentração de cobertura se encontra naqueles municípios que demandam a implementação. Entretanto, quando a variável geográfica é inserida nas análises (Tabela 5), e o foco se concentra naqueles municípios enquadrados nos agrupamentos que apontam uma situação demandante e muito demandante, evidencia-se uma discrepância. Percebe-se que as regiões mais demandantes do programa não são as que têm a maior taxa de cobertura, com destaque para as regiões Nordeste e Norte, que têm taxa de cobertura do programa bem menor quando comparada à região Sudeste. Observa-se outra incoerência: apesar de o Sul possuir baixa taxa de cobertura em 2013, é a região que historicamente tem recebido maior quantidade de recursos do PAA: aproximadamente 34\% do total aplicado no período de 2003 a 2013 (CONAB, 2014). Além disso, Grisa et al. (2010) demons-

Tabela 4. Taxa percentual de cobertura municipal do PAA no Brasil por agrupamento

\begin{tabular}{lcccc}
\hline \multicolumn{1}{c}{ Grupos } & Total & Executou & Não Executou & Taxa de Cobertura \\
\hline Muito Demandante & 794 & 213 & 581 & $27 \%$ \\
Demandante & 1395 & 428 & 967 & $31 \%$ \\
Pouco Demandante & 1495 & 225 & 1270 & $15 \%$ \\
Nada Demandante & 1881 & 223 & 1658 & $12 \%$ \\
Total & 5565 & 1089 & 4476 & $19,57 \%$ \\
\hline
\end{tabular}

Fonte: Resultados da pesquisa.

Tabela 5. Taxa percentual de cobertura do PAA junto aos municípios cujo agrupamento apresentou-se como muito demandante e demandante

\begin{tabular}{lccc}
\hline Região & $\begin{array}{c}\text { Municípios de demanda do } \\
\text { programa }\end{array}$ & $\begin{array}{c}\text { Municípios de demanda com } \\
\text { atuação do programa }\end{array}$ & Taxa percentual de cobertura \\
\hline Sudeste & 202 & 79 & $39 \%$ \\
Nordeste & 1610 & 494 & $31 \%$ \\
Norte & 308 & 59 & $19 \%$ \\
Centro-Oeste & 37 & 6 & $16 \%$ \\
Sul & 32 & 3 & $9 \%$ \\
Total & 2189 & 641 & $29,28 \%$ \\
\hline
\end{tabular}

Fonte: Resultados da pesquisa. 
tram que estados cujos índices de pobreza e insegurança alimentar são mais elevados são beneficiados com menos recursos.

Corroborando com o trabalho de Simão, Silva e Silveira (2014), observou-se que, apesar de parte significativa dos recursos do programa ser alocada junto aos municípios e regiões demandantes, algumas regiões têm sido preteridas. Isso torna-se preocupante, uma vez que a região Norte não alcançou um quinto de sua real demanda e a região Nordeste não alcançou metade da sua demanda no tocante à taxa ideal de cobertura do PAA. Ainda que tenha ocorrido crescimento no número de projetos, de produtores participantes, entidades beneficiadas e valores dos recursos (HESPANHOL, 2013), sua abrangência, medida pela taxa de cobertura, é limitada e concentrada.

Apesar de observar-se uma evolução na dimensão do programa ao longo dos anos e mudança na priorização das regiões atendidas, é flagrante a concentração histórica dos recursos no Sul e no Nordeste. Essa configuração é resultante do fato de que, conjuntamente, elas concentram quase $67 \%$ do total de explorações familiares contabilizadas no último censo agropecuário (SACCO DOS ANJOS e BECKER, 2014). Observa-se que a concentração da distribuição de recursos das políticas públicas voltadas à agricultura familiar não se limita apenas ao PAA. No caso do Pronaf, estudos comprovaram que os estados da região Sul captavam maior volume de recursos (GUANZIROLI, 2007; SOUZA, NEY e PONCIANO, 2011), em detrimento dos estados de regiões de agricultura familiar mais fragilizada, como o Nordeste.

Um programa é considerado menos eficaz quanto mais domicílios/indivíduos do público-alvo não forem contemplados com os benefícios do programa. Muitas vezes, o erro de focalização é inevitável devido a fatores como a imperfeição dos indicadores utilizados para se fazer a seleção e o acesso precário à informação (MIRANDA e COSTA, 2007). No caso do PAA, o erro de focalização está relacionado a limitações na escolha das áreas favorecidas pelo programa. Por regra, é seguido o critério de oferta alimentar e, mais do que tudo, da demanda das organizações proponentes pelo programa, e não de maior retorno social, em que a insegurança alimentar assume maior gravidade e a agricultura familiar é menos consolidada (SPAROVEK et al., 2007; SOARES, BARROS e MAGALHÃES, 2007). Para Sparovek et al. (2007, p. 64), "a falta de um cadas- tro para participação [dos agricultores] no PAA limita a possibilidade de definir critérios (geográficos e socioeconômicos) de forma a priorizar aqueles mais necessitados". Ademais, muitos agricultores são excluídos por não atenderem às exigências do programa (Declaração de Aptidão ao Pronaf - DAP, infraestrutura etc.), sendo estes talvez os que mais precisassem do PAA.

A baixa capacidade de execução no PAA nas regiões Norte e Nordeste está relacionada ao fato de o programa exigir das organizações proponentes elevada contrapartida em organização social, recursos humanos e materiais, uma vez que o programa se expressa nos locais na forma de rede, interligando unidades familiares de produção e estas com associações e cooperativas, poder público, entidades sociais, famílias beneficiadas e todos com instâncias estaduais e com a estrutura nacional do Programa. Estas condições não estão presentes em todas as organizações sociais, agravando-se à medida que o nível de pobreza dos associados se acentue. Por conseguinte, onde o PAA tomaria maior relevância e retorno social é exatamente onde se tem maior dificuldade de execução (GRISA et al., 2010).

Quando o foco de análise passa a ser o grupo de agricultores atendidos pelo programa em 2013, verifica-se que, do total de beneficiários fornecedores participantes, $49 \%$ estão enquadrados nos grupos A, A/C e $B$ do Pronaf, que são os de menores renda, com destaque para a região Nordeste, onde 76\% dos agricultores beneficiários estavam enquadrados no grupo de menor renda. Entretanto, o programa, de modo geral, ainda tem contemplado principalmente os agricultores familiares "mais capitalizados", como já indicaram outras pesquisas (SPAROVEK et al., 2007; SOARES, BARROS e MAGALHÃES, 2007).

A distorção de acesso ao Programa também pode estar relacionada às disparidades no grau de inserção no mercado dos agricultores familiares nas diferentes regiões brasileiras. Buanain (2006) mostra que, na região Sul, quase $70 \%$ dos familiares são considerados integrados ou muito integrados ao mercado. Nas regiões Norte e Nordeste, mais de $50 \%$ dos agricultores foram classificados como pouco integrados ao mercado. Nas unidades minifundistas no Nordeste, faltam condições que as viabilizem como produtores agropecuários. Esses agricultores são também os mais pobres e não conseguem gerar renda suficiente para manter a família acima da linha de pobreza. Na região Norte, a restrição é de acesso aos mercados e não de tama- 
nho de estabelecimento. Portanto, são estas regiões as mais demandantes de políticas públicas com o PAA, que visam integrar os agricultores ao mercado institucional de alimentos, proporcionando geração de renda e segurança alimentar.

\section{Conclusões}

Os resultados obtidos mostraram que o Programa de Aquisição de Alimentos tem sido eficaz apenas na focalização naqueles municípios de maior propensão a sua execução. Porém, sua taxa de cobertura nessas regiões ainda é muito incipiente e desigual, dada as necessidades locais dos municípios. Tal fato demonstra a necessidade de aprimorar a capacidade de discriminar e de selecionar o público-alvo. O Programa também carece de ampliação e alocação regionalizada de suas ações, principalmente nas regiões mais demandantes, caso do Nordeste e Norte. Portanto, este estudo caminha na direção de orientar os gestores públicos a elaborar políticas que visem minimizar essas disparidades, tornando o acesso às políticas públicas mais eficiente e eficaz.

Há a necessidade de estabelecimento de mecanismos que garantam representação regional mais equilibrada das ações do programa, levando em consideração os diferentes aspectos culturais, econômicos e sociais no processo de implementação de políticas públicas. O monitoramento deve ser aplicado de forma mais consistente no nível micro para identificar áreas de intervenção específicas. Para lidar com as disparidades regionais no desempenho de políticas públicas de segurança alimentar e fortalecimento da agricultura familiar, também é necessário o estabelecimento de mecanismos formais que garantam um fluxo de regular de informações e ideias entre os tomadores de decisão em termos nacional, regional, estadual e municipal. O programa ainda carece de avanços em termos de marco legal e de sua institucionalidade, de modo a conferir-lhe todos os atributos de uma política pública no tocante, entre outros, às ações dos vários setores envolvidos e ao montante e regularidade na liberação de recursos.

Denota-se que a criação do mercado de compra institucional do PAA trouxe consigo um vetor impor- tante de mudanças. Ainda que limitado pela baixa cobertura, dimensão do programa e pelo número de agricultores participantes, estudos apontam que o programa tem promovido a geração de renda, a sustentação de preços aos agricultores familiares e contribuído para a segurança alimentar e nutricional da população urbana e rural. Ainda é necessário incrementar o investimento para consolidar e ampliar essas experiências, não somente em relação à quantidade de recursos destinados à continuidade e à ampliação dos projetos existentes, mas, também, tendo em vista a acumulação de experiências para propor a realização de políticas complementares.

Evidencia-se que existe espaço para implementar diferentes políticas de fortalecimento da agricultura familiar e combate à fome e à pobreza nas regiões brasileiras, principalmente nas regiões Norte e Nordeste, onde se concentram os maiores níveis de pobreza do País e onde observa-se que, internamente às regiões, a frequência de pobres é sistematicamente maior no meio rural do que no meio urbano (MONTEIRO, 2003). Desta forma, este estudo mostra a importância de desenvolver, manter e focalizar políticas de combate à fome e a pobreza e de fortalecimento da agricultura familiar, a fim de contribuir com a sua eficácia e efetividade. Por outro lado, torna-se necessária a realização de um processo contínuo de formação dos produtores rurais, que os permita desenvolver suas atividades econômicas e melhorar o acesso a informações sobre o funcionamento das políticas públicas e suas condições. É necessário buscar caminhos que assegurem maior cobertura para alcançar as populações mais pobres, por meio da criação de mecanismos que facilitem o acesso a essas populações.

Como limitações desta pesquisa deve-se destacar que a análise não dá conta de todos os aspectos locais, regionais, estruturais e socioculturais de cada região, no processo de avaliação do PAA. Outro ponto importante se refere ao recorte temporal: a seção cruzada não traz ao pesquisador os fatos ocorridos antes e após o ponto de análise, uma vez que a coleta e a análise dos dados são realizadas em um único ponto do tempo. Esses fatos abrem novas perspectivas de pesquisa, principalmente delineamentos que considerem os aspectos tanto da parte governamental, quanto aqueles relativos às especificidades regionais e locais. 


\section{Referências}

ABRAMOVAY, R. Paradigmas do capitalismo agrário em questão. São Paulo: Hucitec/ANPOCS/UNICAMP, 2007.

ANDREWS, C. W. Anti-poverty policies in Brazil: reviewing the past ten years. International Review of Administrative Sciences, v. 70, n. 3, p. 477-488, 2004.

ARRETCHE, M. Tendências nos Estudos sobre Avaliação. In: RICO, E. (Org.). Avaliação de políticas sociais: uma questão em debate. São Paulo: Cortez, 1998, p. 29-41.

BECKER,C.eSACCODOSANJOS,F.Segurança alimentar e desenvolvimento rural: limites e possibilidades do Programa de Aquisição de Alimentos da Agricultura Familiar (PAA), em municípios do sul gaúcho. Segurança Alimentar e Nutricional, v. 17, p. 61-72, 2010.

BRASIL. MDS. Programa de Aquisição de Alimentos, Brasília, 2015a. Disponível em: < https://goo.gl/ifJgb3>. Acesso em: 8 nov. 2015.

. PAA Data - Dados de 2015, 2015b. Disponível em: <https://goo.gl/HpgL3G>. Acesso: 05 set. 2015.

. Indice de Desenvolvimento da Família (IDF), 2013. Disponível em: < https://goo.gl/2lksnd>. Acesso em: 10 set. 2015.

BUAINAIN, A. M. Agricultura familiar, agroecologia $e$ desenvolvimento sustentável: questões para debate. Brasília: IICA, 2006.

, ROMEIRO, A. e GUANZIROLI, C. E. Agricultura Familiar e novo mundo rural. Sociologias (UFRGS), Porto Alegre, v. 5, n. 10, p. 312-347, 2003.

CARDEN, F. e ALKIN, M. Evaluation roots: an international perspective. Journal of MultiDisciplinary Evaluation, v. 8, n. 17, 2012.

CARVALHO, S. N. Avaliação de programas sociais: balanço das experiências e contribuição para o debate. Revista São Paulo em Perspectiva, v. 17, p. 185-197, 2003.

CASTEL, J. M. et al. Family dairy farms in the Podlasie province, Poland: farm typology according to farming system. Spanish Journal Agricultural Research, v. 8, n. 4, p. 946-961, 2010.

CERQUEIRA, P. S., ROCHA, A. G. e COELHO, V. P. Agricultura familiar e políticas públicas: algumas reflexões sobre o Programa de Aquisição de Alimentos no estado da Bahia. Revista Desenbahia, v. 3, p. 55-78, 2006.

COADY, D., GROSH, M. e HODDINOTT, J. Targeting of transfers in developing countries: review of lessons and experience. Washington, DC: World Bank, IFPRI, 2004.

CONAB. Programa de Aquisição de Alimentos - PAA Resultados das Ações da Conab 2013, 2014. Disponível em < https://goo.gl/Lbp1ce>. Acesso em: 3 set. 2015.
CORDEIRO, A. Resultados do programa de aquisição de alimentos - PAA: a perspectiva dos beneficiários. Brasília: CONAB, 2007.

CORRÊA, F. C. M. Programa de Aquisição de Alimentos (PAA): uma comparação entre dois Estados do Brasil. 2008. 80f. Dissertação (Mestrado em Agronegócios), Faculdade de Agronomia e Medicina Veterinária, UNB, Brasília, 2008.

COSTA, C. C. M. et al. Determinantes do desenvolvimento do setor agropecuário nos municípios. Revista de Administração, São Paulo, v. 48, n. 2, p. 295-309, abr./jun. 2013.

COSTA, F. L. e CASTANHAR, J. C. Avaliação de programas públicos: desafios conceituais e metodológicos. Revista Brasileira de Administração Pública, v. 37, p. 969-992, 2003.

CUNHA, C. G. S. Avaliação de políticas públicas e programas governamentais: tendências recentes e experiências no Brasil. Secretaria de Coordenação e Planejamento/RS, 2006.

DELGADO, G. C. A questão agrária no Brasil, 19502003. Mundo Rural. Sociologias, n. 10, p. 312-347, 2003.

., CONCEIÇÃO, J. C. P. R. D. e OLIVEIRA, J. J. D. Avaliação do programa de aquisição de alimentos da agricultura familiar (PAA). Brasília: IPEA, 2005.

DIAS, T. F. e ROCHA, L. A. O Programa de Aquisição de Alimentos (PAA) da agricultura familiar e seus efeitos nos municípios do Rio Grande do Norte - 2005 a 2011. Administração Pública e Gestão Social, v. 7, n. 1, p. 16-25, 2015.

DORETTO, M. e MICHELLON, E. Avaliação dos impactos econômicos, sociais e culturais do Programa de Aquisição de Alimentos. Sociedade e desenvolvimento rural, v. 1, n. 1, 2007.

FARIA, R. M. Avaliação de programas sociais: evoluções e tendências. In: RICO (Org.). Avaliação de políticas sociais: uma questão em debate. São Paulo: Cortez, 2006.

GARSON, G. D. Statnotes: topics in multivariate analysis [online]. Disponível em: < http://faculty.chass. ncsu.edu/garson/PA765/statnote.htm> Acesso em: 27 nov. 2014.

GIUDICI, P. e FIGINI, S. Applied Data Mining for Business and Industry. 2. ed. Londres: John Wiley \& Sons Ltd, 2009.

GOMES, A. e BASTOS, F. Limites e possibilidades da inserção da agricultura familiar no PAA em Pernambuco. Sociedade e Desenvolvimento Rural, v. 1, n. 1, 2007.

GRAZIANO DA SILVA, J., DEL GROSSI, M. E. e DE FRANÇA, C. G. Fome Zero: la experiencia brasileña. MDA: Brasília, 2012. 
GRISA, C. et al. Contribuições do Programa de Aquisição de Alimentos à segurança alimentar e nutricional e à criação de mercados para a agricultura familiar. Agriculturas, v. 8, n. 3, p. 34-41, 2011.

GRISA, C. e SCHNEIDER, S. Três Gerações de Políticas Públicas para a Agricultura Familiar e Formas de Interação entre Sociedade e Estado no Brasil. Revista de Economia e Sociologia Rural, Piracicaba-SP, v. 52, p. S125-s146, 2014. Suplemento.

GUANZIROLI, C. E. et al. Agricultura familiar e reforma agrária no século XXI. Rio de Janeiro: Garamond, 2001.

GUANZIROLI, C. E. PRONAF dez anos depois: resultados e perspectivas para o desenvolvimento rural. Revista de Economia e Sociologia Rural, v. 45, n. 2, p. 301-328, abr.jun. 2007.

HAIR JUNIOR, J. F. et al. Fundamentos de métodos de pesquisa em administração. Porto Alegre: Bookman, 2005.

HESPANHOL, R. A. M. Programa de Aquisição de Alimentos: limites e potencialidades de políticas de segurança alimentar para a agricultura familiar. Sociedade E Natureza, v. 25, n. 3, p. 469-483, set./dez. 2013.

HOFFMANN, R. Determinantes da insegurança alimentar no Brasil em 2004 e 2009. Segurança Alimentar e Nutricional, v. 20, n. 2, p. 219-235, 2013.

HOFFMANN, R. e KAGEYAMA, A. Pobreza, insegurança alimentar e pluriatividade no Brasil. Teoria e Evidência Econômica, v. 14, p. 9-35, 2007.

IBGE. Censo Agropecuário 2006 - Resultados Preliminares. Disponível em: <https://goo.gl/mKu1rK> . Acesso em: 08 nov. 2015.

. Censo Demográfico 2010. Rio de Janeiro, 2010.

IPC-IG. Structured Demand and Smallholder Farmers in Brazil: the Case of PAA and PNAE. International Policy Centre for Inclusive Growth (IPC - IG) United Nations Development Programme, Brasilia, 2013.

JANNUZZI, P. M. Considerações sobre o uso, mau uso e abuso dos indicadores sociais na formulação e avaliação de políticas públicas municipais. Revista de Administração Pública, Rio de Janeiro, v. 36, n. 1, p. 51-72, jan./fev. 2002.

. Indicadores para diagnóstico, monitoramento e avaliação de programas sociais no Brasil. Revista do Serviço Público, Brasília, v. 56, p. 137-160, 2005.

- Avaliação de programas públicos por meio da análise estruturada dos relatórios de auditoria da Controladoria Geral da União. Cadernos Gestão Pública e Cidadania, São Paulo, v. 16, n. 59, jul./dez. 2011.

KAGEYAMA, A. A., BERGAMASCO, S. M. P. P. e OLIVEIRA, J. T. A. Uma tipologia dos estabelecimentos agropecuários do Brasil a partir do censo de 2006. Revista de Economia e Sociologia Rural, Piracicaba-SP, v. 51, n. 1, p. 105-122, jan./mar. 2013.

KOUTSOU, S., PARTALIDOU, M. e PETROU, M. Present or Absent Farm Heads? A Contemporary Reading of Family Farming in Greece. Sociologia Ruralis, v. 51 , n. 4 , out. 2011.

LEAL, S. C. T. O Programa de Aquisição de Alimentos (PAA) no Pontal do Paranapanema/SP. Revista NERA, v. 18, n. 26, p. 147-164, 2015. Edição Especial.

LEMOS, S. M. e LIMA, I. S. O Programa de Aquisição de Alimentos (PAA) como política pública para a agricultura familiar e o desenvolvimento local. Oikos: Revista Brasileira de Economia Doméstica, Viçosa, v. 25, n. 1, p. 069-092, 2014.

LLANILLO, R. F. et al. Regionalização da agricultura do Estado do Paraná, Brasil. Ciência Rural, n. 36, n. 1, p. 120-127, jan./fev. 2006.

MARQUES, P. E. M., MOAL, M. F. e ANDRADE, A. G. F. Programa de Aquisição de Alimentos (PAA) no estado de São Paulo. Ruris, v. 8, n. 1, p. 63-89, mar. 2014.

MATTEI, L. Programa de Aquisição de Alimentos da Agricultura Familiar (PAA): antecedentes, concepção e composição geral do Programa. Cadernos do CEAM (UnB), v. 7, p. 33-44, 2007.

MATTEI, L. e MALUF, R. Pobreza rural: concepções, determinantes e agenda de políticas públicas para seu enfrentamento. Fórum DRS: Boletim Eletrônico, ano 5, edição 72, jan. 2011.

MILHORANCE, C. e GABAS, J. J. Reframing development from the South? A debate on the internationalization of Brazil's rural policies. In: International Conference of Public Policy, 2015, Milão. Anais..., Milão, 2015.

MIRANDA, C. e COSTA, C. (Orgs.). Ações de combate à pobreza rural: metodologia para avaliação de impactos. Brasília: Instituto Interamericano de Cooperação para a Agricultura (IICA), 2007. (Série Desenvolvimento Rural Sustentável, v. 6).

MONTEIRO, C. A. A dimensão da pobreza, da desnutrição e da fome no Brasil. Estudos Avançados, São Paulo, v. 17, n. 48, p. 7-20, 2003.

MOTA, D. M. et al. As catadoras de mangaba no Programa de Aquisição de Alimentos - PAA: um estudo de caso em Sergipe. Revista de Economia e Sociologia Rural, v. 52, n. 03, p. 241-262, jul./set. 2014.

MÜlLER, A. L., SILVA, M. K. e SCHNEIDER, S. A. A construção de políticas públicas para a agricultura familiar no Brasil: o Programa de Aquisição de 
Alimentos. Estudos Sociedade e Agricultura, v. 20, Rio de Janeiro, 2012.

NUNES, A. e BARROS, E. Caracterização territorial do desemprego registado em Portugal: aplicação da análise de clusters. Economia Global e Gestão, v. 15, n. 3, p. 9-26, 2010.

OLIVEIRA, J. S. et al. Insegurança alimentar e estado nutricional de crianças de São João do Tigre, no semiárido do Nordeste. Revista Brasileira de Epidemiologia, São Paulo, v. 12, n. 3, p. 413-423, 2009.

ORTEGA, A. C., JESUS, C. M. e SÓ, L. L. S. O PAA-leite na Bahia e em Minas Gerais: uma avaliação preliminar de seus modelos de implementação. Cadernos do CEAM, v. 5, n. 24, p. 57-89, ago. 2006.

PEREIRA, M. E. B. G. e LOURENZANI, A. E. B. S. Desafios e perspectivas do programa de aquisição de alimentos no município de Tupã - SP. Ciência e Natura, v. 36, n. 2, 2014.

PNUD. Atlas do Desenvolvimento Humano no Brasil 2013. Disponível em: <http://www.atlasbrasil.org.br/2013/ pt/>. Acesso em: 5 nov. 2015.

REZENDE, L. M. e JANNUZZI, P. M. Monitoramento do Plano de Desenvolvimento da Educação: proposta de aprimoramento do Ideb e de painel de indicadores. Revista do Serviço Pública, Brasília, v. 59, n. 2, p. 121-150, abr./jun. 2008.

SACCO DOS ANJOS, F. e BECKER, C. Agricultura familiar e mercados institucionais: o desenvolvimento como liberdade. Revista Econômica do Nordeste, v. 45, p. 92-101, 2014.

SALGADO, R. J. S. F. e DIAS, M. M. Análise da influência do Programa de Aquisição de Alimentos (PAA) sobre a qualidade de vida de agricultores familiares do município de Viçosa/MG. Revista Perspectivas em Políticas Públicas, v. 6, n. 11, 2013.

SEGALL-CORRÊA, A. M. et al. Transferência de renda e segurança alimentar no Brasil: análise dos dados nacionais. Revista de Nutrição, Campinas, v. 21 (Suplemento), 2008.

SILVA, T. C. R. et al. Entre o Potencial de Transformação Local e os Entraves do Programa de Aquisição de Alimentos na Cidade de Viçosa-MG. Administração Pública e Gestão Social, v. 4, p. 399-419, 2012.

SILVA, T. C., ROSA, P. R. e AMODEO, N. B. P. A importância do Programa de Aquisição de Alimentos (PAA) nos territórios da cidadania dos Estados de Minas Gerais e da Bahia: Criação de novos mercados a partir da visão dos agentes de assistência técnica e extensão rural (ATER). Mundo Agrario, v. 15, n. 29, ago. 2014.

SIMÃO, G. L., SILVA, E. A. e SILVEIRA, S. F. R. Grau de Cobertura do Programa de Aquisição de Alimentos (PAA) Junto aos Agricultores Familiares do Estado de Minas Gerais. Revista de Economia e Sociologia Rural, Piracicaba, v. 52, n. 03, p. 533-548, jul./set. 2014.

SOARES, A., BARROS, A. R. e MAGALHÃES, A. M. Estudo sobre os benefícios do PAA-leite: produtores e consumidores. In: PAES-SOUZA, R. e VAITSMAN, J. Cadernos de estudo: desenvolvimento social em debate: síntese das pesquisas de avaliação de programas sociais do MDS, n. 5, 2007.

SOUZA, P. M. e BARBE, L. C. Desigualdades regionais na distribuição dos financiamentos do Pronaf: uma análise do período de 1998 a 2012. Revista Econômica do Nordeste, v. 45, p. 31-43, 2014. Suplemento.

SOUZA, P. M., NEY, M. G. e PONCIANO, N. J. Evolução da distribuição dos financiamentos do PRONAF entre as Unidades da Federação, no período de 1999 a 2009. Revista Brasileira de Economia, v. 65, p. 303-313, jul./set. 2011.

SPAROVEK, G. et al. Estudo comparativo das diferentes modalidades do PAA - região nordeste. In: PAES-SOUZA, R. e VAITSMAN, J. Cadernos de estudo: desenvolvimento social em debate: síntese das pesquisas de avaliação de programas sociais do MDS, n. 5, 2007.

SWENSSON, L. F. J. Institutional procurement of food from smallholder farmers: the case of Brazil. Food and Agriculture Organization of The United Nations, 2015.

TEIXEIRA, E. C. O papel das políticas públicas no desenvolvimento local e na transformação da realidade. Revista AATR, 2002.

THOMSON, A. e METZ, M. Implications of economic policy for food security: a training manual, 1998. Disponível em: <https://goo.gl/Vkqozd>. Acesso em: 20 nov. 2015.

TRICHES, R. M. e GRISA, C. Entre mudanças e conservadorismos: uma análise dos programas de aquisição de alimentos (PAA e PNAE) a partir da retórica da intransigência. Revista NERA, v. 18, n. 26, p. 10-27, 2015. Edição especial.

TWEETEN, L. The economics of global food security. Review of Agricultural Economics, v. 21, n. 2, p. 473-488, 1999.

VIANNA, R. P. de T. et al. Insegurança alimentar das famílias residentes em municípios do interior do estado da Paraíba, Brasil. Revista de Nutrição, Campinas, v. 21 (Suplemento), 2008. 
